TITLE:

\title{
Analytically solving the relativistic Dirac-Coulomb equation for atoms and molecules
}

AUTHOR(S):

Nakatsuji, H; Nakashima, H

\section{CITATION:}

Nakatsuji, H ... [et al]. Analytically solving the relativistic Dirac-Coulomb equation for atoms and molecules. Physical Review Letters 2005, 95(5): 050407.

\section{ISSUE DATE:}

2005-07-29

URL:

http://hdl.handle.net/2433/39929

RIGHT:

Copyright 2005 American Physical Society 


\title{
Analytically Solving the Relativistic Dirac-Coulomb Equation for Atoms and Molecules
}

\author{
Hiroshi Nakatsuji ${ }^{1,2}$ and Hiroyuki Nakashima ${ }^{1}$ \\ ${ }^{1}$ Department of Synthetic Chemistry and Biological Chemistry, Graduate School of Engineering, Kyoto University, \\ Katsura, Nishikyo-ku, Kyoto 615-8510, Japan \\ ${ }^{2}$ Fukui Institute for Fundamental Chemistry, Kyoto University, 34-4 Takano-Nishihiraki-cho, Sakyo-ku, Kyoto 606-8103, Japan
}

(Received 26 April 2005; published 29 July 2005)

\begin{abstract}
A method of solving the Schrödinger equation in an analytically expanded form reported previously is extended to the relativistic case and a general method of exactly solving the Dirac-Coulomb equation has been proposed for atoms and molecules. Some problems characteristic of the relativistic case are discussed. Test applications to the hydrogenlike, heliumlike atoms are satisfactory, implying a high potentiality of the proposed method also for the relativistic case.
\end{abstract}

DOI: 10.1103/PhysRevLett.95.050407

As noted by Dirac in 1929 [1], the Schrödinger equation (SE) for atoms and molecules,

$$
\begin{gathered}
H \psi=E \psi, \\
H=\sum_{i}-\Delta_{i} / 2-\sum_{i, A} Z_{A} / r_{A i}+\sum_{i>j} 1 / r_{i j}
\end{gathered}
$$

is a major quantum principle governing chemistry. So, establishing a general method of exactly solving the SE is a dream of theoretical chemists. In the SE, the exact wave function $\psi$ is determined by the Hamiltonian $H$, so that the exact $\psi$ may be written as a functional of $H$ applied to some appropriate function $\psi_{0}$ as

$$
\psi=f(H) \psi_{0} .
$$

Recently, we have studied the structure of the exact wave function, i.e., a possible functional form of $f(H)$, and proposed the iterative configuration or composites interaction (ICI) method and the simplest extreme coupled cluster (SECC) method as the methods of constructing the exact wave function [2-4]. Generally, when $f$ is expanded in a Maclaurin series of $H$, it would include higher powers of $H$, but the integrals of the higher powers of $H$ over approximate wave functions do not exist, i.e., diverge because of the singularity of the Coulomb potentials included in the Hamiltonian [4]. Thus, the singularity difficulty commonly occurs in the method of analytically solving the SE of atoms and molecules.

We have proposed two methods of solving the singularity problem, i.e., by introducing the inverse Schrödinger equation (ISE) [3] and the scaled Schrödinger equation (SSE) [4]. Between the two, the latter method was simpler and more general than the former. Combined with the ICI method [2], it opened a new field of calculating the exact wave functions of atoms and molecules in an analytical expansion form [4]. We have applied the method successfully to helium atom and hydrogen molecule [4,5] and further to three-to-five electron atoms [5]: these calculations have given a basis to confirm the high potentiality of the proposed method. A short overview was given as a part of the account of the recent works of one of the authors [6].
PACS numbers: 03.65.Pm, 03.65.Ca, 03.65.Ge, 31.15.-p

Dirac equation and Dirac-Coulomb equation.-When atoms and molecules include heavier elements, the relativistic effect becomes very important and even dominant. For a one-electron system, the Dirac equation (DE) describes exactly the relativistic motion of an electron. It has an explicit Hamiltonian that obeys the Lorentz transformation. For many-electron systems, however, we do not have such relativistic equations that are as compact and accurate as the DE. The Dirac-Coulomb equation (DCE) and the Dirac-Coulomb-Breit equation (DCBE) are approximate and do not exactly obey the Lorentz transformation, but they are expected to be very accurate for atoms and molecules. For more accurate formulations, we use quantum electrodynamics, but there, we do not have explicit Hamiltonians.

The DE for one-electron system and the DCE for $N$ electron system are written as

$$
\hat{H} \psi=E \psi,
$$

where the Hamiltonian $\hat{H}$ is defined by the equations,

$$
\begin{aligned}
\hat{H} & =\sum_{i}^{N} \hat{H}_{N}(i)+\hat{I}_{(N)}\left(\sum_{i<j}^{N} w_{i j}\right), \\
\hat{H}_{N}(i) & =\hat{I}_{(i-1)} \otimes \hat{h}_{i} \otimes \hat{I}_{(N-i)}, \\
\hat{h}_{i} & =\left(\begin{array}{cc}
\left(v_{i}+c^{2}\right) \hat{I}_{2} & c\left(\sigma \cdot \mathbf{p}_{i}\right) \\
c\left(\sigma \cdot \mathbf{p}_{i}\right) & \left(v_{i}-c^{2}\right) \hat{I}_{2}
\end{array}\right),
\end{aligned}
$$

and $w_{i j}=1 / r_{i j} . \hat{I}_{n}$ and $\hat{I}_{(n)}$ are the unit matrices of orders $n$ and $4^{n}$, respectively. $\hat{h}_{i}$ is the $4 \times 4$ matrix one-electron Hamiltonian and $v_{i}=-\Sigma_{A} Z_{A} / r_{A i}$ is the nuclear attraction operator for electron i. $\sigma$ and $\mathbf{p}_{i}$ are the ordinary Pauli spin matrix and the momentum operator of electron $i$. The relativistic Hamiltonian $\hat{H}$ is a $4^{N} \times 4^{N}$ operator matrix and the wave function $\psi$ is a vector composed of $4^{N}$ elements. The term $E \psi$ in Eq. (4) is also written as $E \hat{I}_{(N)} \psi$ : mostly we use the simpler expression. For the one-electron case $(N=1)$, the DCE reduces to the DE. In the nonrelativistic limit, the DCE reduces to the SE. So, the DCE should be very accurate for atoms and molecules. 
In this Letter, our aim is to formulate a general method of solving the "exact" $\psi$ of the DE and DCE in an analytical expansion form.

In the relativistic case, there are no theories dealing with the structure of the exact wave function, nor the theories for constructing the exact wave function. So, we first generalize our theory for the SE to the relativistic case. The Coulomb singularity problem is common to both the nonrelativistic and relativistic cases and is solved by introducing the scaled equations [4]. Another problem that often appears in the relativistic field is the variational instability and collapse. Though some studies using kinetic and other balances $[7,8]$ were reported, there seems to be no established method. It was impressive that Pestka and Karwowski closed their important chapter of the Rychlewski's book in 2003 [7] by noting "The Hylleraas CI approach to solving the Dirac-Coulomb eigenvalue problem is still in its infancy. However, it certainly offers a perspective of highly accurate benchmark results, at least for two-electron systems."

Formulation of the exact wave function. - Now the first step of our project is to clarify the structure of the exact relativistic wave function $\psi$ and then to give a general method of constructing it in an analytical form. We follow the same arguments as in the previous articles [2-4]. First, the variational principle for $\psi$ is written as

$$
\langle\psi|\hat{H}-E| \delta \psi\rangle=0 .
$$

This is a stationary principle for calculating the best possible $\psi$. The $H$-square equation is formulated as

$$
\left\langle\psi\left|(\hat{H}-E)^{2}\right| \psi\right\rangle=0,
$$

and is satisfied only with the exact $\psi$. Then, we have the following theorem.

Theorem: When a $\psi$ includes only one variable matrix $\hat{C}$ (diagonal) with the element $C_{n}\left(n=1 \ldots 4^{N}\right)$ and when an arbitrary variation of $\psi$ satisfies

$$
\delta \psi=\delta \hat{C}(\hat{H}-E) \psi,
$$

then this $\psi$ has the structure of the exact wave function. Proof: When we define $(\hat{H}-E) \psi=\chi, \chi$ is a column vector of the elements $\chi_{n}\left(n=1 \ldots 4^{N}\right)$. When we substitute $\delta \psi$ given by Eq. (8) into the variational principle, Eq. (6), we obtain $0=\langle\psi|(\hat{H}-E) \delta \hat{C}(\hat{H}-E)| \psi\rangle=$ $\sum_{n}^{4^{N}} \delta C_{n}\left\langle\left|\chi_{n}\right|^{2}\right\rangle$. Since $\delta C_{n}$ is arbitrary, this means $\left\langle\left|\chi_{n}\right|^{2}\right\rangle=0$ for all $n$, which is equivalent to Eq. (7). So, among $\psi$ with the form of Eq. (8), variationally optimal wave functions are the exact solutions of the DE and the DCE. Proof ends. This theorem is a generalization of the similar theorem given previously for the nonrelativistic case [2] and the argument is free from the technical arguments like variational collapse, since the $H$-square equation is valid only for the exact wave function.

Based on the above theorem, we propose the relativistic ICI method just as in the nonrelativistic case. We define the simplest ICI (SICI) wave function by the recurrence for- mula,

$$
\psi_{n+1}=\left[1+\hat{C}_{n}\left(\hat{H}-E_{n}\right)\right] \psi_{n},
$$

where $n$ is an iteration number. The variation of $\psi_{n+1}$, $\delta \psi_{n+1}=\delta \hat{C}_{n}\left(\hat{H}-E_{n}\right) \psi_{n}$, becomes equal to that given by Eq. (8) at the convergence where everything becomes $n$ independent, so that the SICI gives the exact wave function at convergence. We can also formulate a more general ICI method by dividing the operator $\hat{H}$ in the $N_{D}$ parts (ICIND) [2], but omit it here for brevity.

The DC Hamiltonian includes the Coulomb potentials that cause the singularity problem. To avoid it, we introduce the scaled DE and DCE that are given by

$$
g\left(\hat{H}-E \hat{I}_{(N)}\right) \psi=0,
$$

where the scaling function $g$ is a positive scalar function of electron coordinates and can become zero only at the singular points $r_{0}$ of the potential $V$ but satisfies $\lim _{r \rightarrow r_{0}} g V \neq 0$. By this introduction of the scaled equation, we can avoid the singularity problem as in the nonrelativistic case [4]. Since this idea is very general, we can use it for singular potentials that appear in other fields of physics. Based on the scaled DCE, we introduce the SICI method that is free from the singularity problem by

$$
\psi_{n+1}=\left[1+\hat{C}_{n} g\left(\hat{H}-E_{n}\right)\right] \psi_{n} .
$$

The SICI includes only one variable matrix $\hat{C}_{n}$ at each iteration and its convergence may be slow [2]. A better performance is expected by introducing the free ICI method as in the nonrelativistic case [4]. In the free ICI, we take all the independent functions $\left\{\phi_{i}\right\}^{n}$ from the righthand side of Eq. (11) and give independent coefficients to all such functions $\phi_{i}$, i.e.,

$$
\psi_{n+1}=\sum_{i}^{\text {all }} c_{i} \phi_{i}
$$

From the variational point of view, this free ICI should converge faster than the SICI. Note that $\phi_{i}$ is a column vector of $4^{N}$ dimension and $c_{i}$ is a diagonal matrix with $4^{N}$ elements. In the free ICI, each iteration does not depend on the results of the former iterations and so no accumulation of error occurs. In some choice of $g$, the right-hand side of Eq. (11) may include diverging functions but they are eliminated in the free ICI process since such functions are inappropriate for describing the wave function.

Variational calculations. - Now, we have an analytical function having the structure of the exact wave function in the form of free ICI [Eq. (12)]. The next step is the variational calculation of the variables $\left\{c_{i}\right\}$ and the corresponding energy, which we call the diagonalization step. Here, an obstacle that often appears in the relativistic field is the so-called variational collapse. Since the lowest electronic state of the DE and the DCE is not the lowest state of these equations, the so-called Ritz-type property does not hold. However, a method to recover Ritz-type property was 
proposed by Hill and Krauthauser [9] for one-electron DC calculations with basis set expansion method. They introduced the inverse Hamiltonian and wrote the DE as

$$
\hat{H}^{-1} \psi=E^{-1} \psi \text {. }
$$

As in our case of the inverse Schrödinger equation [3], it is easy to show the equivalence between the original equation and the inverse one. For the inverse DE, the electronic ground state is the highest solution against the complete vacuum and therefore we have the Ritz-like variational principle $\tilde{E}^{-1}=\left\langle\tilde{\psi}\left|\hat{H}^{-1}\right| \tilde{\psi}\right\rangle /\langle\tilde{\psi} \mid \tilde{\psi}\rangle \leq E_{0}^{-1}$, where $\tilde{\psi}$ is a variational trial function for $\psi$ and $E_{0}$ is the true energy of the ground electronic state. Now, how do we explicitly write down the inverse Hamiltonian? A clever trick [9] is to take our variational function in the form of $\tilde{\psi}=\hat{H} \varphi$ with $\varphi$ representing a free variation. Then, the above variational equation is rewritten as

$$
\tilde{E}^{-1}=\langle\varphi|\hat{H}| \varphi\rangle /\left\langle\varphi\left|\hat{H}^{2}\right| \varphi\right\rangle \leq E_{0}^{-1},
$$

and the variance is formulated as

$$
\begin{aligned}
\sigma^{2}= & \left\langle\tilde{\psi}\left|\left(\hat{H}^{-1}-E^{-1}\right)^{2}\right| \tilde{\psi}\right\rangle /\langle\tilde{\psi} \mid \tilde{\psi}\rangle \\
= & \left(\langle\varphi \mid \varphi\rangle-2 E^{-1}\langle\varphi|\hat{H}| \varphi\rangle\right. \\
& \left.+E^{-2}\left\langle\varphi\left|\hat{H}^{2}\right| \varphi\right\rangle\right) /\left\langle\varphi\left|\hat{H}^{2}\right| \varphi\right\rangle
\end{aligned}
$$

which we can calculate. For the exact wave function, $\sigma^{2}$ must be zero: this is the inverse $H$-square theorem [3].

At the diagonalization step of the relativistic free ICI, we utilize the above Ritz-like variational equation to ensure the bound property. Though it was introduced originally for the one-electron DE [9], we utilize it for solving the many-electron DCE. We know from the theory of ICI that our free ICI function $\varphi_{n}$ approaches the exact wave function, and therefore it is easy to show that our variational function $\tilde{\psi}_{n}$ also approaches the exact wave function. Since $\hat{H}$ must not be singular to define its inverse, we often have to shift it by a constant $W$, i.e., $\hat{H}^{\prime}=\hat{H}+W \hat{I}_{(N)}$. Further, since Eq. (14) includes the integral of $\hat{H}^{2}$ that is more strongly singular than $\hat{H}$, we have to eliminate a larger number of diverging functions from our ICI basis than in the ordinary case that involves only $\hat{H}$. This may make the inverse variation method rather slowly converging. Nevertheless, the bound property of this variational method is useful in actual calculations where we do not know the true energy $E_{0}$. In the ICI formulation, the excited states are calculated as the higher solutions: an appropriate choice of $\psi_{0}$ is necessary for obtaining a reasonable convergence speed.

Another method of avoiding the variational collapse is to ensure kinetic balance, etc. on the basis functions $[7,8,10]$. From the DE, we obtain a relation connecting small and large components as $\phi^{S}=\left[\left(c \sigma \mathbf{p} /\left(E-V+c^{2}\right)\right] \phi^{L}\right.$. A strict use of this relation is called atomic balance and an approximate one kinetic balance. We note here that the balancing similar to the atomic balance is automatically done in the ICI formalism. In ICI, $\psi_{n+1}$ is generated by applying the DC operator to $\psi_{n}$ [Eq. (11)] and this is essentially a balancing that is theoretically valid even for many-electron systems. We call this balancing ICI balance. In an earlier stage of the ICI calculation, the number of the basis functions is small and the ICI balancing would be insufficient. So, practically, we have to do an additional balancing before diagonalization. Detailed examinations of such balancing methods will be given in the forthcoming paper.

Hydrogen isoelectronic atoms. - The first application of the present theory is to hydrogen isoelectronic atoms for which the exact analytical wave functions are known. We take $H(Z=1)$ and $\mathrm{Fe}^{25+}(Z=26)$ : the relativistic effect is larger for the latter. For the ground state of these oneelectron atoms, the DE is written with the radial part alone, separated from the angular part, as [11]

$$
\left(\begin{array}{cc}
c^{2}-Z / r & c[-d / d r-(1-k) / r] \\
c[d / d r+(1+k) / r] & -c^{2}-Z / r
\end{array}\right) \psi=E \psi,
$$

where $k= \pm[j+(1 / 2)]$ with $j$ being the quantum number of the total angular momentum and $c=137.03596$ [12]. The relativistic exact wave function has a mild singularity at the position of the nucleus [11].

In the free ICI, we took the starting wave function as $\psi_{0}^{L}=\psi_{0}^{S}=\exp (-\alpha r)$ with $\alpha=1.5 Z$ instead of $\alpha=Z$. The scaling function was taken to be $g=r^{99 / 100}$ instead of $g=r$ in order to describe, ad hoc, the mild singularity. The free ICI iteration process is shown in Table I. The varia-

TABLE I. Relativistic free ICI energy (a.u.) of hydrogenlike atoms ( $Z=1$ and 26). ${ }^{a}$

\begin{tabular}{lccccc}
\hline \hline$n^{\mathrm{b}}$ & $M^{\mathrm{c}}$ & Energy $(\mathrm{H})$ & $\operatorname{Energy}\left(\mathrm{Fe}^{25+}\right)$ & $\Delta^{L}\left(\mathrm{Fe}^{25+}\right)$ & $\Delta^{S}\left(\mathrm{Fe}^{25+}\right)$ \\
\hline 0 & 2 & -0.375033696 & -268.305491 & $3.3 \times 10^{-1}$ & $3.3 \times 10^{-1}$ \\
1 & 6 & -0.493381703 & -336.985794 & $9.8 \times 10^{-2}$ & $7.1 \times 10^{-2}$ \\
2 & 12 & -0.499822155 & -340.983740 & $1.6 \times 10^{-2}$ & $1.1 \times 10^{-2}$ \\
3 & 20 & -0.500004337 & -341.096421 & $1.6 \times 10^{-3}$ & $1.2 \times 10^{-3}$ \\
4 & 30 & -0.500006642 & -341.097830 & $1.2 \times 10^{-4}$ & $9.0 \times 10^{-5}$ \\
5 & 42 & -0.500006657 & -341.097839 & $7.2 \times 10^{-6}$ & $5.3 \times 10^{-6}$ \\
& -0.500006657 & -341.097839 & 0.0 & 0.0 \\
\hline \hline
\end{tabular}

${ }^{\mathrm{a}}$ The bottom row shows the exact values.

${ }^{b}$ Iteration number.

${ }^{\mathrm{c}}$ Number of the independent functions for expanding $\psi$. Half and half for the large and small components. 
tional method was an ordinary one. For both $\mathrm{H}$ and $\mathrm{Fe}^{25+}$, the energy at fifth iteration agrees with the exact value to nine figures. $\Delta$ defined by $\left[\int\left|\psi_{n}^{\mathrm{ICI}}-\psi_{\text {exact }}\right|^{2} d \tau\right]^{1 / 2}$ approaches zero for both large and small components as shown for heavier $\mathrm{Fe}^{25+}$ : the ICI wave function itself also converges to the exact one. We have performed many different calculations, but as far as we do ICI, we have never experienced the variational collapse for the oneelectron case. It was unnecessary to resort to the inverse Hamiltonian as Hill et al. considered originally [9].

Helium isoelectronic atoms. - The next application is to heliumlike two-electron atoms, whose DCE is the matrix equation of dimension 16. We calculated $\mathrm{He}(Z=2)$ and $\mathrm{Th}^{88+}(Z=90)$. The wave function $\psi$ is composed of $\psi^{l l}$, $\psi^{l s}, \psi^{s l}$, and $\psi^{s s}$, each being 4 -vector. They satisfy $\psi^{l l}(1,2)=-\psi^{l l}(2,1), \psi^{l s}(1,2)=-\psi^{s l}(2,1)$, and $\psi^{s s}(1,2)=$ $-\psi^{s s}(2,1): \psi^{l s}$ and $\psi^{s l}$ are not independent. The initial function was taken as $\psi_{0}^{l l}=A\left(s_{1 / 2,1 / 2} \otimes s_{1 / 2,-1 / 2}\right), \psi_{0}^{l s}=$ $s_{1 / 2,1 / 2} \otimes p_{1 / 2,-1 / 2}-s_{1 / 2,-1 / 2} \otimes p_{1 / 2,1 / 2}, \quad$ and $\quad \psi_{0}^{s s}=$ $A\left(p_{1 / 2,1 / 2} \otimes p_{1 / 2,-1 / 2}\right)$ with $A$ being an antisymmetrizer, and a function $\chi=r^{\gamma-1} \exp (-Z r)$ was used with $\gamma=$ $\left[k^{2}-(Z / c)^{2}\right]^{1 / 2}$ that describes a mild singularity at the nucleus. We performed the free ICI using the $g$ function, $g=1+r_{1}+r_{2}+r_{12}$, and therefore, we dropped out from our expansion bases the functions that lead to diverging integrals. We used the inverse Hamiltonian method at the diagonalization step to ensure the bound property. We used $W=c$ to avoid the singularity originating from the ls part.

Table II shows the result. When we used the inverse Hamiltonian at the diagonalization step, the ground state energy monotonically converged from above (actually, the inverse energy converged from below) toward the literature value $[8,12]$ for both $Z=2$ and 90 . For $Z=2$, the energy of Ref. [12] is more accurate than that of Ref. [8]: the former included $r_{12}$ explicitly, but the latter did not. For $Z=90$, the value cited [8] is due to the calculation not including $r_{12}$ explicitly. Since we used the inverse Hamiltonian, the present energy given in Table II should be an upper bound of the true energy. When we used the regular Hamiltonian, instead of the inverse Hamiltonian, at the diagonalization step, the variational collapse occurred for both $Z=2$ and 90 .

Conclusion. - We have proposed in this Letter a method of solving the DCE for atoms and molecules in an analytical expansion form. The relativistic ICI automatically generates the wave function having the exact structure. The exact ICI balancing is done in the iteration process. For the one-electron atoms, we experienced no variational problem as far as we used ICI. For the two-electron atoms, we could avoid the variational collapse by introducing the inverse Hamiltonian. Thus, we propose the relativistic free ICI method based on Eqs. (11) and (12) and the inverse variational method having the Ritz-like property as a general method of calculating the exact solutions of the DCE of many-electron atoms and molecules. This Ritz-like
TABLE II. Relativistic free ICI energy (a.u.) of the heliumlike atoms $(Z=2$ and 90$)$ using the inverse Hamiltonian at the diagonalization step.

\begin{tabular}{lrll}
\hline \hline \multicolumn{1}{c}{$n^{\mathrm{a}}$} & $M^{\mathrm{b}}$ & \multicolumn{1}{c}{ Energy $(\mathrm{He})$} & \multicolumn{1}{c}{ Energy $\left(\mathrm{Th}^{88+}\right)$} \\
\hline 0 & 3 & -2.75008563 & -9166.575433 \\
1 & 12 & -2.88771973 & -9166.809415 \\
2 & 38 & -2.90307277 & -9166.858084 \\
3 & 105 & -2.90375350 & -9166.881423 \\
4 & 224 & -2.90384265 & -9166.894218 \\
5 & 482 & -2.90385116 & -9166.903050 \\
6 & 852 & -2.90385405 & -9166.907227 \\
Ref. [8] & & -2.902636 & -9166.9272 \\
Ref. [12] & & -2.903857 & \\
\hline \hline
\end{tabular}

${ }^{\mathrm{a}}$ Iteration number.

${ }^{\mathrm{b}}$ Number of the independent functions for expanding $\psi$. More details of $M\left(M_{l l}, M_{l s}, M_{s s}\right)$ are $3(1,1,1), 12(4,4,4), 38(12$, $13,13), 105(29,35,41), 224(66,69,89), 482(145,139,198)$, and $852(257,223,342)$.

property is useful in actual calculations where we do not know the true energy $E_{0}$.

Our method of calculating the analytical exact wave functions has been confirmed to have a high potentiality for solving the SE of atoms and molecules [4-6] and extended here to solve the relativistic DCE. We have still many things to do in future to develop this new methodology as a really useful method in quantum chemistry.

This study has been supported financially by a Grant for Creative Scientific Research from the Ministry of Education, Science, Culture, and Sports of Japan.

[1] P. A. M. Dirac, Proc. R. Soc. A 123, 714 (1929).

[2] H. Nakatsuji, J. Chem. Phys. 113, 2949 (2000); H. Nakatsuji and E. R. Davidson, ibid. 115, 2000 (2001); H. Nakatsuji, ibid. 115, 2465 (2001); H. Nakatsuji, ibid. 116, 1811 (2002); H. Nakatsuji and M. Ehara, ibid. 117, 9 (2002); H. Nakatsuji and M. Ehara, ibid. 122, 194108 (2005).

[3] H. Nakatsuji, Phys. Rev. A 65, 052122 (2002).

[4] H. Nakatsuji, Phys. Rev. Lett. 93, 030403 (2004).

[5] H. Nakatsuji, H. Nakashima, and Y. Kurokawa (to be published).

[6] H. Nakatsuji, Bull. Chem. Soc. Jpn. (to be published).

[7] G. Pestka and J. Karwowski, in Explicitly Correlated Wave Functions in Chemistry and Physics - Theory and Applications, edited by J. Rychlewski (Kluwer, Dordrecht, 2003), pp. 331-346.

[8] A. Kolakowska, J.D. Talman, and K. Aashamar, Phys. Rev. A 53, 168 (1996).

[9] R. N. Hill and C. Krauthauser, Phys. Rev. Lett. 72, 2151 (1994).

[10] G. Pestka, Phys. Scr. 69, 203 (2004).

[11] J. J. Sakurai, Advanced Quantum Mechanics (AddisonWesley, Reading, MA, 1967).

[12] A. Kolakowska, J. Phys. B 30, 2773 (1997). 